\title{
Restoration of antegrade ejaculation after transurethral bladder neck injection of Deflux for retrograde ejaculation: a case report of natural conception
}

\author{
Hu Han', Sai Liu', Xiaoning Liang ${ }^{2}$, Hong'en Lei ${ }^{1}$, Gao Li ${ }^{1}$, Jianzhong Zhang ${ }^{1}$, Peng Zhang ${ }^{1}$, \\ Xiaodong Zhang ${ }^{1}$, Long Tian ${ }^{1}$ \\ ${ }^{1}$ Institute of Urology, Capital Medical University; Department of Urology, Beijing Chao-Yang Hospital, Capital Medical University, Beijing, China; \\ ${ }^{2}$ Department of Ultrasound, Beijing Chao-Yang Hospital, Capital Medical University, Beijing, China \\ Correspondence to: Long Tian. Department of Urology, Beijing Chao-Yang Hospital, 8 Gongren Tiyuchang Nanlu, Chaoyang District, Beijing \\ 100020, China. Email: longtian@ccmu.edu.cn.
}

\begin{abstract}
Retrograde ejaculation contributes to male infertility. Pharmacotherapy has a limited role in retrograde ejaculation. Deflux, a viscous substance, consists of two components: dextranomer microspheres and stabilized hyaluronic acid for the treatment of children with vesicoureteral reflux. According to the published articles, the authors described the first case achieved not only restoration of antegrade ejaculation but also followed conception of the spouse after transurethral bladder neck injection of Deflux. A 30-yearold male kept infertile after 3 years of marriage. The patient reported that he could not ejaculate in an antegrade direction after puberty when he reached sexual climax. There was no semen expulsed from the urethral meatus. Medicine prescribed by a local hospital didn't restore normal ejaculation. Four embryos fertilized by intracytoplasmic sperm injection developed well until transplantation, but none survived. The local reproductive center confirmed that his spouse preserved full capacity of fertility. He was diagnosed as retrograde ejaculation by real-time monitoring of transrectal ultrasound. He received transurethral bladder neck injection of Deflux. One month later, the patient restored antegrade ejaculation and the spouse of the patient conceived. During 18 months of follow-up, no related complications of the surgery have been observed. Transurethral bladder neck injection of Deflux can be considered as an alternative treatment method for retrograde ejaculation, and natural conception is possible thereafter.
\end{abstract}

Keywords: Case repot; Deflux; ejaculation; pregnancy; urinary bladder

Submitted Feb 17, 2020. Accepted for publication Aug 28, 2020.

doi: 10.21037/tau-20-593

View this article at: http://dx.doi.org/10.21037/tau-20-593

\section{Introduction}

Retrograde ejaculation is the complete or partial inability to ejaculate in an antegrade direction, and the sperm enters the bladder through the neck of the bladder in the reverse direction (1). Not only can it cause male infertility, but it can also lead to decreased sexual satisfaction. Pharmacotherapy for retrograde ejaculation is limited, and surgical intervention is rare $(2,3)$. This article first reports a patient with idiopathic retrograde ejaculation not only restored antegrade ejaculation, but also successfully fathered a child naturally after transurethral bladder neck injection of Deflux. According to previous reports, antegrade ejaculation can be achieved (4-6). But whether the patients with restoration of antegrade ejaculation can achieve natural conception has not been answered. This case achieved natural conception answered this question and makes transurethral bladder neck injection of Deflux more valuable in dealing retrograde ejaculation. We present the following article in accordance with the CARE Guideline. We present the following article in accordance with the CARE reporting checklist (available at http://dx.doi. org/10.21037/tau-20-593). 


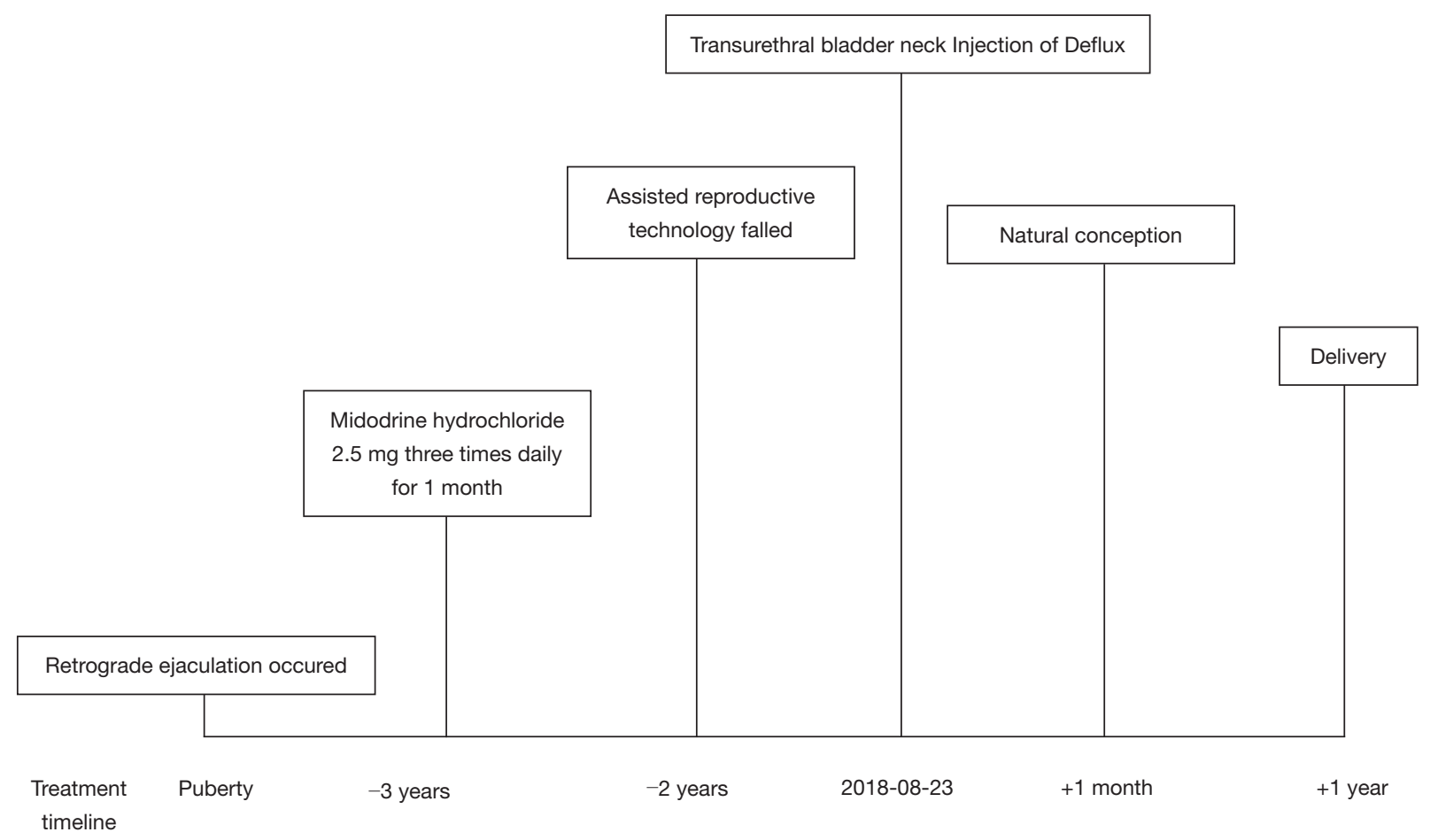

Figure 1 Timeline of history.

\section{Case presentation}

A 30-year-old male was admitted to the hospital with chief complaint of infertility for 3 years. The patient reported that he could not ejaculate in an antegrade direction after puberty when he reached sexual climax, as displayed in Figure 1. There was no semen expulsed from the urethral meatus. Midodrine hydrochloride $2.5 \mathrm{mg}$ three times daily for one month prescribed by a local hospital didn't restore normal ejaculation. And midodrine hydrochloride discontinued because the patient was anxious to influence of sperm quality affected by medicine. Sperm has been retrieved in the urine after ejaculation with sodium bicarbonate orally taken. Four embryos fertilized by intracytoplasmic sperm injection developed well until transplantation. Two embryos at a time were transplanted once and again, but none survived. The local reproductive center confirmed that his spouse preserved full capacity of fertility. For further treatment, the patient visited and was admitted to our hospital. The patient denied the past history of diabetes, surgery, trauma, or mental disorders.

Physical examination indicated no obvious structural abnormality of the external genitalia. Normal parameters have been detected in blood routine, blood biochemistry, reproductive hormones. Real-time transrectal ultrasound indicated semen was retrogradely ejaculated into the bladder after emission, as was seen in Figure $2 \mathrm{~A}$.

According to the published articles, injection of Deflux, a viscous collagen, to the bladder neck may restore antegrade ejaculation for the patients with retrograde ejaculation. The patient received transurethral bladder neck injection of Deflux with informed consent and comprehensive communication to the surgeons before the surgery, as was seen in Figure 2B. A total of two $\mathrm{mL}$ Deflux was injected equally into the bladder neck at clock positions of 2, 4, 6, 8 and 10 o'clock under cystoscopy in 5 minutes. The needle tip was completely immersed into the submucosa. Mucosal bulge was observed during injection. In case of urinary retention, a urinary catheter was placed and removed one day later. In follow-up of 18 months, the patient converted to antegrade ejaculation with high satisfaction 1 month after the surgery without lower urinary tract symptoms or other complications. Natural conception of the spouse occurred one month after the surgery.

All procedures performed in studies involving human participants were in accordance with the ethical standards of the institutional and/or national research committee(s) and with the Helsinki Declaration (as revised in 2013). Written 

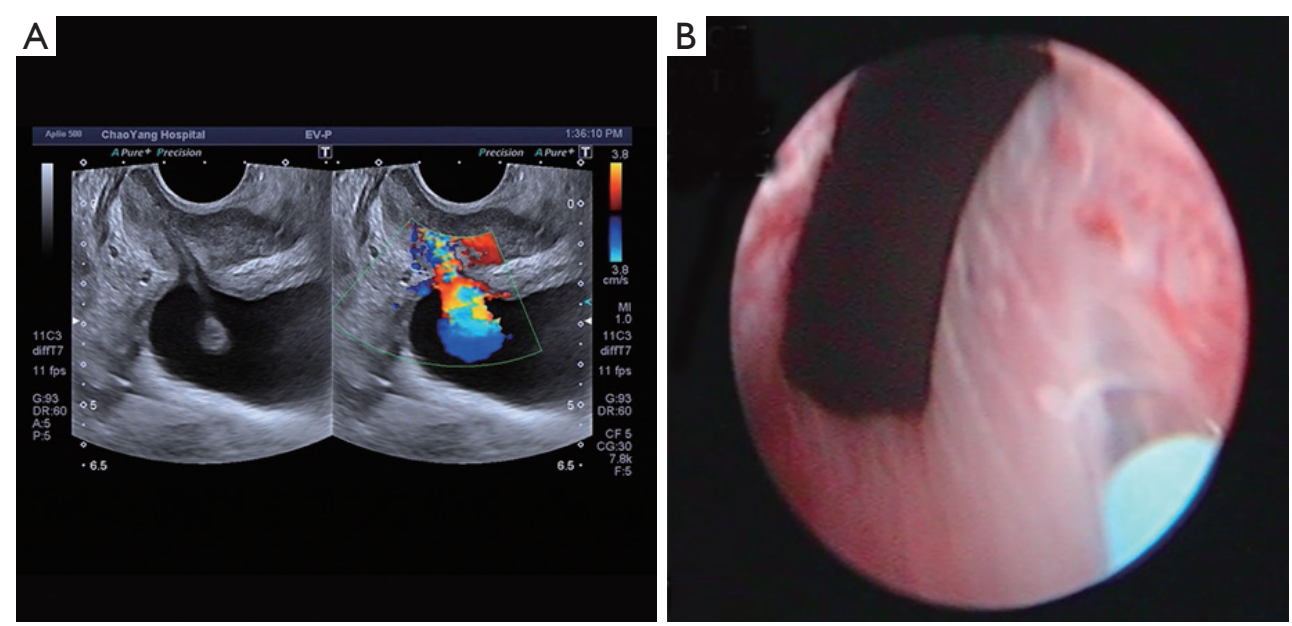

Figure 2 Preoperative ultrasound image and operative image. (A) Semen was ejaculated into the bladder under transrectal ultrasound. (B) Transurethral bladder neck injection of Deflux.

informed consent was obtained from the patient.

\section{Discussion}

The normal ejaculation process includes two parts, emission, and ejaculation. During this process, the closure of bladder neck is necessary to avoid semen from flowing or ejaculating into the bladder. If the bladder neck is not fully closed, the semen will enter the bladder in the reverse direction (7). The diagnosis of retrograde ejaculation is mainly determined by the presence of sperm after ejaculation in urine (1), but there are reports that sperm also could be found in urine after ejaculation in $73 \%$ of males with normal fertility (8). This phenomenon challenges the notion to set presence of sperm after ejaculation as a criterion for retrograde ejaculation. In order to evaluate the ejaculation in this case, transrectal ultrasound has been applied to monitor the ejaculation process in real time. The ultrasound clearly showed that the bladder neck kept open during emission and ejaculation, and semen was ejaculated from the posterior urethral to the bladder in color Doppler flow imaging. Figure 1 is consistent with a previous report of ultrasound diagnosis of retrograde ejaculation $(9,10)$. Compared with the presence or absence of sperm in the urine after ejaculation, real-time ultrasound can not only differentiate anejaculation, antegrade ejaculation, and retrograde ejaculation, but also monitor the state of the urethral sphincter and bladder neck during retrograde ejaculation, which could pave the way to select a targeted treatment.
Open bladder neck can be observed under cystoscopy. A needle was inserted retrogradely and $2 \mathrm{~mL}$ Deflux was injected submucosally through the needle to five points of the bladder neck equally. Previous literatures reported that $6 \mathrm{~mL}$ collagen injected to 3 positions of the bladder neck (9), $4 \mathrm{~mL}$ collagen to 3 positions (4) or unknown amount of collagen type II to $3-5$ positions (5).

The causes of retrograde ejaculation mainly include congenital malformation, spinal cord injury, retroperitoneal lymph node dissection, diabetes mellitus, bladder neck surgery, and idiopathic ones (8). Reynolds et al. (6) first reported in 1998 that the bladder neck submucosal collagen injection successfully converts retrograde ejaculation to antegrade ejaculation. The retrograde ejaculation of the patient was secondary to bladder neck plastic surgery. Nagai et al. (9) reported the first case of retrograde ejaculation due to incomplete spinal cord injury successfully treated with collagen injection to bladder neck guided by transrectal ultrasound before and after the surgery in 2004. For retrograde ejaculation caused by type I diabetes, transurethral bladder neck injection of type II collagen also has a significant effect (5). Combined this case of idiopathic retrograde ejaculation with literatures, transurethral bladder neck injection of Deflux may become standard treatment in the near future for retrograde ejaculation due to various causes, including but not limited to idiopathic retrograde ejaculation, spinal cord injury, diabetes mellitus, and bladder neck surgery. This treatment can bring new hope for patients with retrograde ejaculation.

During literature review, only three articles reported 
successful conversion of antegrade ejection of the patient with retrograde ejaculation after collagen injection into the urethra and bladder neck (4-6). Reynolds et al. (6) reported that retrograde ejaculation was converted successfully after bladder neck injection of collagen. The patient chose to achieved conception by intrauterine insemination. Kurbatov et al. (5) reported that 2 of 12 patients had restored antegrade ejaculation after collagen injection into the bladder neck, and the control group received saline injection achieved no conception. A study by Hara et al. (4) showed that antegrade ejaculation occurred 1 week after collagen injection in the bladder neck, which gradually decreased over time, and returned to retrograde ejaculation after six months. Unfortunately, during this process, natural conception didn't occur. In this case, the patient restored antegrade ejaculation about 4 weeks after Deflux injection. The spouse has naturally conceived at the same week, and gave birth to a female infant, 11 months after the surgery. This patient of retrograde ejaculation demonstrated that transurethral bladder neck injection of Deflux can be considered as an alternative treatment method for retrograde ejaculation, and natural conception is possible thereafter. Deflux, a viscous substance, consist of glycan anhydride microspheres and stable hyaluronic acid. It is applied to treat vesicoureteral reflux in children with exact effectiveness. It has been reported that Deflux remained to exist around the ureteral orifice in $92 \%$ patients after injection during mean follow-up duration of 22 months (11). The authors analyzed that this successful case benefit from Deflux, a more stable substance than collagen, and five points injection equally positioned around the bladder neck (2, 4, 6, 8, 10 o'clock), compared to injection at 3 positions (4, 6, 8 o'clock) (4,6) and (2, 6, 10 o'clock) (5). Because there are few patients with retrograde ejaculation, transurethral bladder neck injection of Deflux needs further evaluation, including optimal surgical points and number, dose of Deflux, injection depth and other detailed surgical techniques of the surgery.

Until currently, no literature on retrograde ejaculation after bladder neck injection has been published in China. This is the fourth report of transurethral bladder neck injection to deal with retrograde ejaculation in the world, and the first one achieved natural conception after bladder neck injection (4-6). Deflux is first applied by the authors for retrograde ejaculation, while collagen was applied in the first two cases published. Compared with side effects of long-term pharmacotherapy for retrograde ejaculation, such as anxiety, depression and so on, there are few related complications of transurethral bladder neck injection of Deflux (5). After removal of the urinary catheter, no complications observed. In these 3 studies, fourteen patients restored antegrade ejaculation, while none of them have fathered naturally (4-6). Incomplete antegrade ejaculation or poor sperm activity might contribute to infertility of the 12 patients. And the fertility of spouses has not been evaluated. Transurethral bladder neck injection of Deflux, a minimally invasive technique, can make natural conception possible, save medical expenses, and avoid female discomfort of assisted reproductive technology. There are a few limitations in this technique. As a transurethral technique, it may induce lower urinary tract symptoms. Complications and clinical efficacy need to be further evaluated in longterm follow-up.

In conclusion, transurethral bladder neck injection of collagen or Deflux for retrograde ejaculation is in an exploratory stage. Although there is a successful case report, randomized controlled trials in large sample size are needed to further evaluate the treatment.

\section{Acknowledgments}

Funding: None.

\section{Footnote}

Reporting Checklist: The authors have completed the CARE reporting checklist. Available at http://dx.doi.org/10.21037/ tau-20-593

Peer Review File: Available at http://dx.doi.org/10.21037/tau20-593

Conflicts of Interest: All authors have completed the ICMJE uniform disclosure form (available at http://dx.doi. org/10.21037/tau-20-593). The authors have no conflicts of interest to declare.

Ethical Statement: The authors are accountable for all aspects of the work in ensuring that questions related to the accuracy or integrity of any part of the work are appropriately investigated and resolved. All procedures performed in studies involving human participants were in accordance with the ethical standards of the institutional and/or national research committee(s) and with the Helsinki Declaration (as revised in 2013). Written informed consent was obtained from the patient. 
Open Access Statement: This is an Open Access article distributed in accordance with the Creative Commons Attribution-NonCommercial-NoDerivs 4.0 International License (CC BY-NC-ND 4.0), which permits the noncommercial replication and distribution of the article with the strict proviso that no changes or edits are made and the original work is properly cited (including links to both the formal publication through the relevant DOI and the license). See: https://creativecommons.org/licenses/by-nc-nd/4.0/.

\section{References}

1. Colpi G, Weidner W, Jungwirth A, et al. EAU guidelines on ejaculatory dysfunction. Eur Urol 2004;46:555-8.

2. McMahon CG. Management of ejaculatory dysfunction. Intern Med J 2014;44:124-31.

3. Jefferys A, Siassakos D, Wardle P. The management of retrograde ejaculation: a systematic review and update. Fertil Steril 2012;97:306-12.

4. Hara R, Nagai A, Fujii T, et al. Practical application of color Doppler ultrasonography in patients with ejaculatory dysfunction. Int J Urol 2015;22:609-11.

5. Kurbatov D, Russo GI, Galstyan GR, et al. Correction of

Cite this article as: Han $\mathrm{H}$, Liu S, Liang $\mathrm{X}$, Lei H, Li G, Zhang J, Zhang P, Zhang X, Tian L. Restoration of antegrade ejaculation after transurethral bladder neck injection of Deflux for retrograde ejaculation: a case report of natural conception. Transl Androl Urol 2020;9(5):2270-2274. doi:10.21037/tau-20-593
Retrograde Ejaculation in Patients with Diabetes Mellitus Using Endourethral Collagen Injection: Preliminary Results. J Sex Med 2015;12:2126-9.

6. Reynolds JC, McCall A, Kim ED, et al. Bladder neck collagen injection restores antegrade ejaculation after bladder neck surgery. J Urol 1998;159:1303.

7. Shoshany O, Abhyankar N, Elyaguov J, et al. Efficacy of treatment with pseudoephedrine in men with retrograde ejaculation. Andrology 2017;5:744-8.

8. Sigman M, Boyle K, Jarow JP. Prevalence of sperm in the post-ejaculatory urine of fertile and subfertile men. Urology 2008;71:110-2.

9. Nagai A, Nasu Y, Watanabe M, et al. Analysis of retrograde ejaculation using color Doppler ultrasonography before and after transurethral collagen injection. Int J Impot Res 2004;16:456-8.

10. Nagai A, Watanabe M, Nasu Y, et al. Analysis of human ejaculation using color Doppler ultrasonography: a comparison between antegrade and retrograde ejaculation. Urology 2005;65:365-8.

11. Stenberg A, Larsson E, Läckgren G. Endoscopic treatment with dextranomer-hyaluronic acid for vesicoureteral reflux: histological findings. J Urol 2003;169:1109-13. 